\title{
Application of Markov Chain to the Prediction of Consumer Price Index Song Qianhong*
}

College of Science, Heilongjiang Bayi Agricultural University, Daqing163000, China

DOI: $10.36347 /$ sipms.2020.v07i07.002

| Received: 30.06 .2020 | Accepted: 07.07.2020 | Published: 11.07.2020

*Corresponding author: Song Qianhong

Abstract

Review Article

This paper mainly uses the prediction method of Markov chain to predict the consumer price index. Taking the national consumer price index data from 1989 to 2005 as an example, this paper forecasts the consumer price index in three steps to illustrate the specific application of Markov chain method.

Keywords: Markov chain; Consumer price index; Transfer matrix.

Copyright @ 2020: This is an open-access article distributed under the terms of the Creative Commons Attribution license which permits unrestricted use, distribution, and reproduction in any medium for non-commercial use (NonCommercial, or CC-BY-NC) provided the original author and source are credited.

\section{INTRODUCTION}

Consumer price index $[1,2]$ refers to the price of consumer goods and services purchased by residents. It is closely related to people's life and plays an important role in the price system of the whole national economy. It is an important index compiled by the Statistics Department of our government. In this paper, Markov chain is used to predict consumer price index.

\section{The Establishment of Markov Chain Model}

Markov process is a kind of random process without aftereffect. The object of markov chain prediction is the dynamic system with random change. It predicts the future development of the system according to the transition probability between states [3]. The specific steps of using Markov chain method to predict consumer price index are as follows:

- Step-1 State division: According to the relationship between CPI and inflation rate, CPI is divided into several states;

- Step-2 Establish the transition probability matrix: According to the statistics of the results obtained in Step1, the transition probability matrix of Markov chains with different delay times (step sizes) can be obtained. The formula of calculating the state transition probability matrix is $P_{i j}=N_{i j} / N_{i}$, $i, j=1,2, \cdots, s, P_{i j}$ is the probability of going from state $E_{i}$ to state $E_{j}$ in one step, $N_{i}$ is the number of occurrences of state $E_{i}, N_{i j}$ is the number of times that state $E_{i}$ transits to state $E_{j}$ in one step. Thus, the one-step state transition probability matrix is obtained as $\mathrm{P}$, then the n-step transition probability matrix is $P^{(n)}=P^{n}$;

- Step-3 Predict the state probability of the national consumer price index for the year:

$$
P_{i}^{(k)}, i \in E, k=1,2, \cdots, m
$$

\section{The Example Analysis}

In this paper, taking the national consumer price index data of China from 1989 to 2005 as an example, Markov chain prediction is carried out to illustrate the specific application of Markov chain method and test it. Data of CPI are listed in Table-1.

Table-1: CPI and it's states

\begin{tabular}{|l|c|c|c|c|c|c|c|c|c|c|}
\hline Years & $\mathbf{1 9 8 9}$ & $\mathbf{1 9 9 0}$ & $\mathbf{1 9 9 1}$ & $\mathbf{1 9 9 2}$ & $\mathbf{1 9 9 3}$ & $\mathbf{1 9 9 4}$ & $\mathbf{1 9 9 5}$ & $\mathbf{1 9 9 6}$ & $\mathbf{1 9 9 7}$ & $\mathbf{1 9 9 8}$ \\
\hline Price Index & 118.0 & 103.1 & 103.4 & 106.4 & 114.7 & 124.1 & 117.1 & 108.3 & 102.8 & 99.2 \\
\hline Status & 4 & 3 & 3 & 4 & 4 & 4 & 4 & 4 & 2 & 1 \\
\hline
\end{tabular}

\begin{tabular}{|l|c|c|c|c|c|c|c|}
\hline Years & $\mathbf{1 9 9 9}$ & $\mathbf{2 0 0 0}$ & $\mathbf{2 0 0 1}$ & $\mathbf{2 0 0 2}$ & $\mathbf{2 0 0 0 3}$ & $\mathbf{2 0 0 4}$ & $\mathbf{2 0 0 5}$ \\
\hline Price Index & 98.6 & 100.4 & 100.7 & 99.2 & 101.2 & 103.9 & 101.8 \\
\hline Status & 1 & 2 & 2 & 1 & 2 & 3 & 2 \\
\hline
\end{tabular}




\section{Step-1 State division}

According to the relationship between consumer price index and inflation rate, the national consumer price index from 1989 to 2005 is divided into four state spaces, namely deflation, normal, inflation and serious inflation, as shown in Table 2.

Table-2: The state classification of CPI

\begin{tabular}{|l|l|l|}
\hline State & Level & Interval \\
\hline 1 & Deflation & $x<100$ \\
\hline 2 & Normal & $100 \leq x<103$ \\
\hline 3 & Inflation & $103 \leq x<105$ \\
\hline 4 & Serious inflation & $x>105$ \\
\hline
\end{tabular}

According to the classification standard established in Table-2, the status of the data series of CPI in each year is determined as shown in Table- 1 .

$$
\begin{aligned}
P^{(1)} & =\left[\begin{array}{llll}
0.3333 & 0.6667 & 0 & 0 \\
0.3333 & 0.3333 & 0.3333 & 0 \\
0 & 0.3333 & 0.3333 & 0.3333 \\
0 & 0.1667 & 0.1667 & 0.6667
\end{array}\right] \\
P^{(2)} & =\left[\begin{array}{llll}
0.3333 & 0.4444 & 0.2222 & 0 \\
0.2222 & 0.4444 & 0.2222 & 0.1111 \\
0.1111 & 0.2778 & 0.2778 & 0.3333 \\
0.0556 & 0.2222 & 0.2222 & 0.5000
\end{array}\right] \\
P^{(3)} & =\left[\begin{array}{llll}
0.2593 & 0.4444 & 0.2222 & 0.0741 \\
0.2222 & 0.3889 & 0.2407 & 0.1481 \\
0.1296 & 0.3148 & 0.2407 & 0.3148 \\
0.0926 & 0.2685 & 0.2315 & 0.4074
\end{array}\right] \\
P^{(4)} & =\left[\begin{array}{llll}
0.2346 & 0.4074 & 0.2346 & 0.1235 \\
0.2037 & 0.3827 & 0.2346 & 0.1790 \\
0.1481 & 0.3241 & 0.2377 & 0.2901 \\
0.1204 & 0.2963 & 0.2346 & 0.3488
\end{array}\right]
\end{aligned}
$$

\section{Step-3 Forecast the state of 2006 National Consumer} Price Index

According to the CPI in 2005, 2004, 2003 and 2002 and the corresponding state transition probability

\section{Step-2 Establish the transition probability matrix}

According to the corresponding states of each year in Table-1, according to the calculation of transfer probability matrix, the transfer probability matrix of each step can be obtained as follows:

Table-3: The CPI prediction of 2006

\begin{tabular}{|c|c|c|c|c|c|c|c|}
\hline Initial year & status & Time lag/year & $i=\mathbf{1}$ & $i=\mathbf{2}$ & $i=\mathbf{3}$ & $i=\mathbf{4}$ & Probability source \\
\hline 2005 & 2 & 1 & 0.3333 & 0.3333 & 0.3333 & 0 & $P^{(1)}$ \\
\hline 2004 & 3 & 2 & 0.1111 & 0.2778 & 0.2778 & 0.3333 & $P^{(2)}$ \\
\hline 2003 & 2 & 3 & 0.2222 & 0.3889 & 0.2407 & 0.1481 & $P^{(3)}$ \\
\hline 2002 & 1 & 4 & 0.2346 & 0.4074 & 0.2346 & 0.1235 & $P^{(4)}$ \\
\hline$P_{i}=\sum_{k=1}^{m} P_{i}^{(k)}, i \in E$ & & & 0.9012 & 1.4074 & 1.0864 & 0.6049 & \\
\hline
\end{tabular}


According to Table-3, $\max \left\{P_{i}, i \in E\right\}=1.4074$ and at this point $i=2$. It can be seen that the corresponding state of the CPI in 2006 is 2 (normal), that is, the CPI in that year is between 100 and 103, which is a normal year, which is consistent with the actual value of 101.5.

\section{CONCLUSION}

At present, there are many forecasting methods in economic forecasting, such as markov chain method based on absolute distribution, superposition Markov chain forecasting method, etc. Compared with them, Markov chain has the characteristics of wide forecasting range, high reliability and reasonable and sufficient information utilization. Through the example analysis, it can be seen that the results predicted by the Markov chain model are scientific and reliable, and have strong application value $[4,5]$.

\section{REFERENCES}

1. LI S. Applied Statistics [M]. Bei Jing: Qing Hua University Press, 2005.

2. Mei-Lin Z. Applied Statistics [M]. Shang Hai: Fu Dan University Press, 2004.

3. Er-Hua, Y, De-Ping Z. Probability theory and stochastic processes [M]. Bei Jing: Science Press, 2006.

4. Zhao L, Hong-Ke X. Traffic accident prediction based on Improved Grey Markov chain model [J]. Mathematics in Practice and Theory, 2013; (10):99-98.

5. Zhang R, Le-tian X. Application of Grey Markov chain model in rainfall forecast $[\mathrm{J}]$. Journal of Chongqing University of Technology, 2012; (10):103-1064. 